\section{Reminder to deposit DNA sequences}

As members of the Advisory

Committee to the International

Nucleotide Sequence Database Collaboration (INSDC), which includes the DNA Data Bank of Japan (DDBJ), European Nucleotide Archive (ENA) and GenBank databases, we wish to remind the research community of the importance of depositing complete DNA-sequence data in these databases on publication of their results (see also

S. L. Salzberg et al. Science http://dx.doi.org/10.1126/ science.aaf7672; 2016). Indeed, most journals demand a database accession number as a condition of publication.

Access to the INSDC's databases is free and unrestricted (G. Cochrane et al. Nucleic Acids Res. 44 (D1), D48-D50; 2016), enabling researchers to plan experiments and to analyse existing data. As original contributions, deposited data form part of the scientific record and are citable in the literature. Authors can also correct and update their data: these amended records may be removed from the next database release, but still remain permanently available by accession number.

The INSDC has also created major new repositories for large data collections, notably the Sequence Read Archive at the National Center for Biotechnology Information (NCBI), the DDBJ Sequence Read Archive and the ENA at the European Molecular Biology Laboratory's European Bioinformatics Institute (EMBLEBI). These repositories archive raw data from sequencing experiments, a crucial facility for reproducibility and reuse.

For papers dependent on sequence data from human subjects, unrestricted data release may not be possible. In these cases, we would encourage journal editors to insist on data sharing through other repositories that are not part of the INSDC, such as the NCBI's Database of Genotypes and Phenotypes, EMBL-EBI's European Genome-phenome Archive or DDBJ's Japanese Genotype-phenotype Archive. Steven L. Salzberg ${ }^{\star}$ Johns Hopkins University, Baltimore, USA. salzberg@jhu.edu

${ }^{*}$ On behalf of 9 correspondents (see go.nature.com/tvllbd for full list).

\section{El Niño dons winter disguise as La Niña}

Seasonal weather forecasting relies almost exclusively on El Niño, the climate phenomenon associated with warming in the equatorial Pacific Ocean. When the strongest El Niño on record developed last autumn, it offered an opportunity to showcase decades of investment and advances in long-range forecasting (see Nature 529, 267-268; 2016). Surprisingly, however, actual winter weather events were the opposite of those predicted.

For example, southern California's winter was more about heatwaves and wildfires than deluges; Seattle in Washington endured the wettest winter on record rather than a worsening drought; and the upper Mississippi valley experienced flooding to an extent that had previously occurred only in summer.

El Niño should strengthen the side of the jet stream that is nearer to the Equator, bringing wet weather to the southwest United States and cool temperatures to the southeast. Instead, the side nearest to the pole strengthened. This brought weather that would be more expected of La Niña, the opposite phase of the Southern Oscillation that results from cooling waters.

I suggest considering this inaccurate El Niño forecast in the wider context of the Arctic's influence. Low Arctic sea ice and high Eurasian snow cover this autumn increased a Siberian high-pressure system and heat transport towards the North Pole, weakening the polar vortex this winter (see J. Cohen et al. Nature Geosci. 7, 627-637; 2014). The atmospheric responses to Arctic 'amplification' were better predicted than were those to El Niño outside the tropics (see www.aer.com/winter2016). Judah Cohen Atmospheric and Environmental Research, Lexington, Massachusetts, USA. jcohen@aer.com

\section{Reform oversight of Italy's science funds}

We wish to highlight the stark contrast between the hardship in Italy's publicly funded research community (see G. Parisi Nature 530, 33; 2016) and the reportedly largely unused sums of money allocated to a single research institute over the past 12 years.

After 3 years without any financial provision for bottom-up research, Italy's government is providing $€ 31$ million (US\$36 million) a year for the next 3 years to cover research in the humanities as well as science. Of this year's 4,431 grant applications for this modest sum, just 300-500 will be successful.

By contrast, the government plans to inject $€ 150$ million a year for the next 10 years into the Human Technopole project, which will focus on genomics, big data, ageing and nutrition. The recipient is the Italian Institute of Technology in Genoa, which is self-governing and so not publicly accountable - despite the large sums of public money involved. A recent report indicates that half of the institute's funds remained unspent in 2010-14 (see go.nature.com/ vouywf; in Italian).

The government should establish an adequately funded agency that has transparent jurisdiction over the funding and execution of research. The agency would also monitor the progress of the Human Technopole and oversee its accountability. (See also go.nature.com/hgfplj.)
Ernesto Carafoli ${ }^{\star}$ Venetian Institute of Molecular Medicine, Padua, Italy.

Cesare Montecucco ${ }^{\star}$ University of Padua, Italy.

ernestocarafoli@gmail.com ${ }^{*}$ Supported by 13 signatories (see go.nature.com/3xxovu for full list).

\section{Regulate devices for brain stimulation}

We are concerned that the rapid development and increased accessibility of non-invasive technologies with alleged brain-enhancing capabilities is allowing commercial interest to outpace regulatory mechanisms (see Nature 531, 283-284; 2016 and Nature 531, S6-S8; 2016).

Only limited technical ability is required to build a brain-stimulation device at home - or to dress it up and market it commercially. Even though electrical current can endanger cardiovascular and neural function (see go.nature. com/ej3kgx), there are currently no requirements for safety or efficacy testing of home-use devices through clinical-style trials. Beyond the safety of the devices themselves, the impact of regular or sustained personal use of brain stimulators is unknown.

The public may not appreciate that companies are subject to a strict regulatory framework if their product claims to help an individual to achieve normal function (that is, a treatment), but not if it is sold to enhance function. We urge governments to align their regulatory standards for both applications. Olivia Carter, Jason Forte University of Melbourne, Australia. ocarter@unimelb.edu.au

CONTRIBUTIONS

Correspondence may be sent to correspondence@ nature.com after consulting the guidelines at http:// go.nature.com/cmchno. 